\title{
FAREWELL TO MIRJANA LEGAC - MARINE BIOLOGIST AND MUSEUM CURATOR
}

\section{Dušan ZavodniK}

Vitomira Širole-Paje 6, HR-52210 Rovinj, Croatia (zavodnik.dusan@gmail.com)

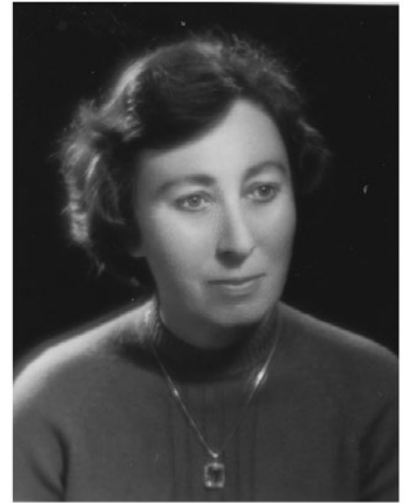

\section{INTRODUCTION}

Our dear friend and colleague, Mirjana Legac, left us forever on November 3, 2018. She was born in 1929, in the picturesque village of Baška located on hilly north-eastern Krk Island. Her birthplace determined her love of the natural beauties of the sea, islands, and the north Adriatic highlands (VILIčić, 2015). From the early youth she showed a keen interest in natural sciences. She was a professional marine biologist and active field researcher, with a BSc degree in biology from the University of Zagreb. She was employed in 1958 as a museum curator at the Natural History Museum Rijeka (Аrко-Pijevac, 2001). Her museum task was to care for various zoological collections, inventorying museum specimens and putting up museum exhibitions as well as workshops, teaching students (Kovačić, 2006; LegAC, 2012; VILIČIć, 2015). From 1970 Mrs Legac was engaged in the duties of senior museum curator and from 1980 those of museum advisor. In two periods she was also an acting director of the Natural History Museum Rijeka (1963 - 1964, 1992 - 1995). She held the position of museum advisor when she retired. From 1961 she collected and worked on the malacofauna of the northern Adriatic and in 1965 she founded the Malacological Collection of the Northern Adriatic. In 2012 she published a valuable catalogue of her museum collection (LEGAC, 2012).

In this farewell only original scientific papers, reviews, and professional papers by Mrs Legac concerning the marine issues of he eastern Adriatic are 
cited. Another of Mirjana's interests was adventures in the shallow Adriatic north-eastern coastal waters, skin diving, and in the inland areas, skiing, hiking and mountain climbing (Legac, 1974 a, b, 2018; Zavodnik et al., 2005; Hemar, 2018). She was an active member of the hiking, mountaineering and speleological community in Croatia from as early as 1948, when she became a member of the Platak Croatian Mountaineering Club (subsequently renamed the Rijeka Club). She participated in organizing excursions, teaching in mountaineering courses, building mountain huts; she climbed the Dolomites, Mt Triglav, Mt Vesuvius, Mt Etna, Mt Olympus, the High Tatras, the High Atlas Mountains in Africa, visited Lake Titicaca in South America (Hemar, 2018). During the Croatian Homeland war she organized the collection of humanitarian help for refugee children.

Mrs. Legac was equally successful in her professional career, both in biology and in the museum fields. She was an esteemed expert in the shellfish and cephalopods of the Adriatic Sea fauna, with emphasis on biocoenological studies within island and channel ecosystems. In addition, one of her most interesting and complicated engagements was research into the habitat and behaviour of the decapod crustacean the Norwegian lobster (Nephrops norvegicus) well studied in the Adriatic a century ago (Gauss-Garrady, 1912; Pesta, 1918). Distribution of this species in the northern part of Croatian Adriatic area was discussed later by Alfirević et al. (1969), Gamulin-Brida et al. (1971), Pérès \& Gamulin-Brida, 1973; ŠTEVČić, 1979, 1990, 1998, and Alfirević (1980). Special field surveys were carried out in 1967-1971 by the research vessel Bios of the Institute for Oceanography and Fisheries Split (Fig. 1). The task concerning benthos communities was supervised by Helena Gamulin-Brida. Besides bottom trawl collections at 6 sites, Petersen grab

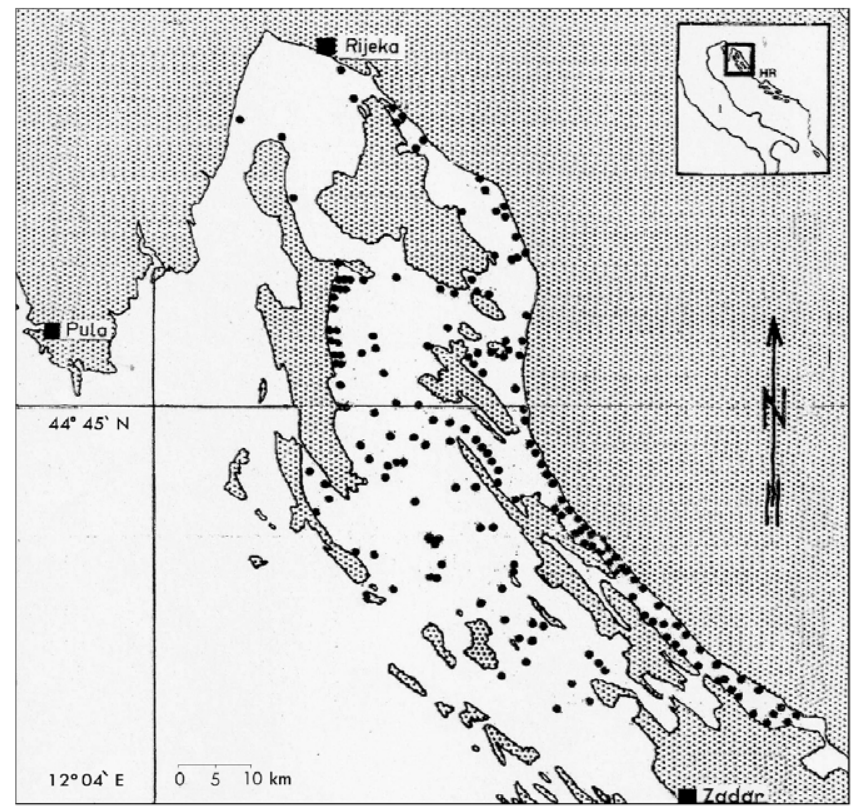

Fig. 1. Surveys for scampi or Norway lobster (Nephrops norvegicus) distribution in the north-eastern Adriatic Sea (1967-1971, by the RV 'Bios'). Sites of grab sampling are displayed. 
$0.2 \mathrm{~m}^{2}$ sampling was accomplished along 73 transects at 188 stations, about $500 \mathrm{~m}$ apart, at 36-105 metres depth (Fig. 2) (Alfirević, 1968; LegaC \& Hrs-Brenko, 1982; VILIČıć, 2015). This was an enormous task extending to the area of Novigradsko more (Novigrad Bay), not repeated in the area until present time. Meanwhile, for various reasons, apart from the bivalve molluscs, rather few accounts on grab sampling results were presented in scientific literature sources (Mollusca Polyplacophora and Scaphopoda: LegAC, 2012 a, b; Gastropoda: LEGAC \& JAKLIN, 2012; Polychaeta: Bellan, 1976 and Amoureux, 1979; Crustacea Decapoda: Števčić, 1979, 1990, 1998; Sipuncula (Murina \& Zavodnik, 1979, 1985/86); Brachiopoda: Legac, 2012 d; Echinodermata (Zavodnik, 1979).

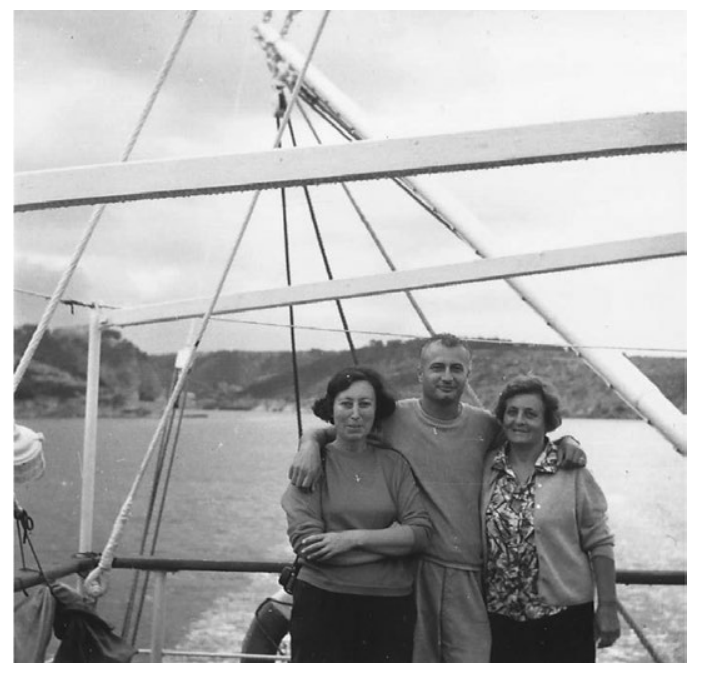

Fig. 2. Field team engaged in marine avertebrate studies. From left to right: museum curator Mirjana Legac, Dr. sc. Dušan Zavodnik and Prof. Dr. sc. Helena GamulinBrida. Photographed in Novigradsko more (Novigrad Bay) on RV 'Bios', on August 31 ${ }^{\text {th }}$, 1971 (author unknown).

One can consider the engagements of Mirjana Legac in biological studies of benthos on Rab Island (LEGAC, 1987) to be the groundwork for modern field research methods applied in the north Adriatic insular region. Subsequently, many localities were surveyed at Lošinj, Krk, and Pag Islands, and nearby islets and cliffs (LEGAC \& Žutić-Maloseja, 1980; Legac \& Legac, 1989; Legac \& Mienis, 2010; Novosel et al., 2002; ZAvoDNiK et al., 2005, 2006; ZAvoDNIK \& LEGAC, 2017). The research results of Mirjana Legac and colleagues were classified and published as original scientific and review papers, or they were presented at professional meetings.

The scientific bibliography on marine issues by the zoological curator Mirjana Legac is presented below. Moreover, her legacy includes some incomplete manuscripts on eastern Adriatic sea fauna. We hope they will be continued shortly. As a marine biology expert, she collaborated in many radio and TV programmes. Besides many scientific and professional papers, many of which were published in Natura Croatica, Mirjana published numerous popular articles about marine biology and protection of nature. For her exceptional museum work in collecting, protecting, her scientific work and museum presentation in natural history, in 1991 she was awarded the Pavao Ritter Vitezović Lifetime Achievement Prize from the Croatian Museum Society. 


\section{ACKNOWLEDGEMENTS}

I am most grateful to Milvana Arko-Pijevac, Mirjana Hrs-Brenko, Virna Brumnić, Vesna Štamol, Marcelo Kovačić, Branko Lenić and Marijana Vuković for precious additional information on bibliographic tasks. Opinions and suggestions by all colleagues are acknowledged. Technical editing of the manuscript was done by Pamela Budicin.

\section{MIRJANA LEGAC - BIBLIOGRAPHY}

Original scientific and review papers

Gamulin-Brida, H., Alfirević, S., Crnković, D., Šimunović, A., Jukić, S., Požar, A. \& Legac, M., 1971: Contribution à l'étude de la dynamique de certaines communautés benthiques en Adriatique. Thalassia Jugosl. 7 (1), 57-66.

Gamulin-Brida, H., Crnković, D., Špan, A., Požar-Domac, A., Jukić, S., Šimunović, A. \& Legac, M., 1977: Expériences et résultats des récherches pluriannuelles sur les biocoenoses benthiques des surfaces de l'Adriatique. Ekologija 12 (2), 115-127.

Gamulin-Brida, H., Ilijanić, V. \& Legac, M., 1977: Contribution à la connaissance des Céphalopodes de l'Adriatique avec égard spécial aux espèces rares ou moins connues. Rapp. Com. Int. Mer Médit. 24 (5), 47-49.

Hrs-Brenko, M. \& Legac, M., 1991: A review of bivalve species in the eastern Adriatic Sea. I. Protobranchia (Solenomyidae, Nuculidae, Nuculanidae). Acta Adriat. 32 (2), 655-670.

Hrs-Brenko, M. \& Legac, M., 1992: Prilozi morskoj flori i fauni lošinske otočne skupine. IV. Školjkaši (Bivalvia). Otočki ljetopis Cres-Lošinj 8, 229-238.

Hrs-Brenko, M. \& Legac, M., 1995: A review of bivalve species in the eastern Adriatic Sea. II. Pteriomorpha (Arcidae and Noetidae). Nat. Croat. 5 (3), 221-247.

Hrs-Brenko, M. \& Legac, M., 1999: A review of bivalve species in the eastern Adriatic Sea. III. Pteriomorpha (Glycimeridae). Nat. Croat. 8 (1), 9-27.

Hrs-Brenko, M. \& Legac, M., 2006: Inter- and intra-species relationships of sessile bivalves on the eastern coast of the Adriatic Sea. Nat. Croat. 15 (4), 203-230.

Hrs-Brenko, M., Legac, M. \& Arko-Pijevac, M., 1998: Contribution to the marine fauna of the Rijeka Bay (Adriatic Sea). 3. Bivalvia. In: Arko-Pijevac M., Kovačıć M. \& Crnković D. (Eds). Zbornik „Prirodoslovna istraživanja riječkog područja“, Prirodoslovni muzej Rijeka, Rijeka, 583-607.

Hrs-Brenko, M., Legac, M. \& Medaković, D., 1989: Varijabilnost morfoloških karakteristika ljuštura roda Cerastoderma Poli, 1795 (Mollusca, Bivalvia, Cardiidae). Biosistematika, 15 (2), 133-148.

Kovač-Konrad, P., BuzjaK, N., KLjaković-GašPić, F. \& Legac, M., 2012: Speleološka, geomorfološka i biospeleološka istraživanja špilje Medove buže na otoku Rabu. In: ANDRIć J. \& LončARIć R. (Eds), Rapski zbornik II., Ogranak Matice Hrvatske u Rabu, Sveučilište u Zadru, Grad Rab i Općina Lopar, Rab, 573-586.

Legac, M., 1964: Prilog poznavanju Cephalopoda kanalskog područja sjevernog Jadrana. Acta Adriat. 11 (25), 181-188.

LeGAC, M., 1969: Some observations on the building up of hiding-places by the Octopus vulgaris Lam. in the channel region of the north-eastern Adriatic. Thalassia Jugosl. 5, 193-200.

LegAc, M., 1974 a: Prilog poznavanju litoralne flore i faune otoka Raba. Vijesti muzeal. konzerv. Hrvatske 23 (5-6), 75-87.

Legac, M., 1974 b: Problem zaštite pješčanih plaža otoka Raba. Vijesti muzeal. konzerv. Hrvatske 23 (5-6), 108-110.

Legac, M., 1974 c: Glavonošci (Cephalopoda) u koćarskim lovinama sjevernog Jadrana. Acta Adriat. 6, 201-206.

LEGAC, M., 1987: Školjkaši (Bivalvia) otoka Raba. Rapski zbornik, JAZU i Skupština općine Rab (Eds), Zagreb, 117-124.

LEGAC, M., 1990: Ugroženost pješčanih plaža i faune školjkaša prekomjernim vađenjem pijeska upriobalju otoka Raba. Ekol. Glasnik 9-10, 9-12. 
LegaC, M., 1991: Revizija zbirke školjkaša (Bivalvia) Brusinina suradnika don Blaža Cvitanovića. Zadarska revija 4-5 (Brusinin zbornik), 81-98.

Legac, M., 1992: Prilozi morskoj flori i fauni lošinjske otočne skupine. V. Glavonošci (Cephalopoda). Otočki ljetopis 8, 239-245.

Legac, M., 1993: Prilog poznavanju školjkaša Nuculana illirica Carrozza, 1987, spec. nov. u Jadranu. IV Kongres biologa Hrvatske, Zagreb, studeni 1993. Book of Abstracts, 387-388.

Legac, M., 2003: Prilog poznavanju školjkaša (Bivalvia) novigradskog područja. Pomorski zbornik 41 (1), 209-21.

Legac, M., 2012 a: Mnogoljušturaši (Polyplacophora) i koponošci (Scaphopoda) u malakološkoj zbirci Prirodoslovnog muzeja Rijeka. In: M. Arko-Pijevac \& B. Surina (Eds), Zbornik Prirodoslovna istraživanja riječkog područja II. Prirodoslovni muzej Rijeka, Rijeka, 315-322.

Legac, M., 2012 b: Revizija malakološke zbirke Prirodoslovnog muzeja Rijeka. In: Arko-Pijevac M . \& Surina B. (Eds), Zbornik Prirodoslovna istraživanja riječkog područja II. Prirodoslovni muzej Rijeka, Rijeka, 323-330.

Legac, M., 2012 c: Katalog zbirke Jadranskih i slatkovodnih školjkaša (Mollusca, Bivalvia) Prirodoslovnog muzeja Rijeka. Prirodoslovna biblioteka 17. The catalogue for the collection of Adriatic and freshwater bivalves (Mollusca, Bivalvia) in Natural History Museum Rijeka. Natural History Library 17. Prirodoslovni muzej Rijeka, Rijeka, 184 pp.

LEgAC, M., 2012 d: New records of recent Brachiopoda from the eastern part of the northern Adriatic Sea. Nat. Croat. 21 (1), 255-258.

Legac, M., 2012 e: New findings of Lutraria angustior Philippi, 1884 and Lutraria oblonga (Gmelin, 1791) on the eastern coast of the Adriatic Sea. Nat. Croat. 15 (4), 203-230.

Legac, M. \& Fabijanić, I., 1994: Contribution to knowledge on the bivalve Glycymeris bimaculata (Poli, 1795) in Pag Bay. Period biol. 96 (4), 450-451.

Legac, M. \& Hrs-Brenko, M., 1975: Sistematska obrada školjkaša (Bivalvia) i glavonožaca (Cephalopoda). Muzeologija 18, 100-105.

Legac, M. \& Hrs-Brenko, M., 1982: A contribution to the knowledge of bivalve species distribution in the insular zones of the northern and part of the middle Adriatic Sea. Acta Adriat. 23 (1/2), 197-225.

Legac, M. \& Hrs-Brenko, M., 1999: A review of bivalve species in the eastern Adriatic Sea. III. Pteriomorphia (Glycymeridae). Nat. Croat. 8 (1), 9-25.

Legac, M. \& Hrs-Brenko, M., 2000: Prvi nalaz školjkaša Rhomboidella prideauxi (Leach, 1815) i Gonilia calliglypta (Dall, 1903) u istočnom dijelu Jadranskog mora. In: Ljubešı́ć N. (Ed.), Zbornik sažetaka priopćenja 7. Hrvatskog biološkog kongresa, Hvar 24-29.9.2000. 'MD' Zagreb (257), 324.

Legac, M. \& Hrs-Brenko, M., 2003: First occurrence of Rhomboidella prideauxi (Leach, 1815) (Mollusca, Bivalvia) in the eastern Adriatic Sea. Nat. Croat. 12 (4), 245-247.

Legac, M. \& JaKlin, A., 2012: Prilog poznavanju morskih puževa (Gastropoda) otoka Raba. Rapski zbornik 2, 511-523.

LegaC, M. \& LegaC, I., 1979: Prilog poznavanju podvodnih speleoloških objekata uz fotodokumentaciju. Simpozij o fotodokumentaciji krasa in jam. Postojna 12.-14.5.1978. Zbornik, 23-26.

Legac, M. \& Legac, I., 1989: Amphoras - an interesting biotope of various flora and fauna species. Period. biol. 91 (1), 122-123.

Legac, M. \& Legac, I., 1998: Prilozi morskoj fauni Riječkog zaljeva. 4. Glavonošci (Cephalopoda). In: Arko-Pijevac M., Kovačıć M. \& Crnković D. (Eds). Prirodoslovna istraživanja riječkog područja. Prirodoslovni muzej Rijeka, Rijeka, 609-616.

Legac, M. \& Mienis, H.K., 2010: New findings of Janthina globosa Swainson, 1822 (Mollusca, Gastropoda) in the eastern part of the Adriatic Sea. Nat. Croat. 19 (1), 245-248.

Legac, M. \& Zavodnik, D., 2017: Morska fauna otoka Paga. In: Znanstveni skup „Pag u prošlosti i sadašnjosti“, 21-23. listopada 1993, Pag i Novalja. Š. BAtović (Ed.), Paški zbornik (2015), 59-78.

Legac, M. \& Žutić-Maloseja, Ž., 1980: Prilog poznavanju bentosa infralitorala u području jugozapadne obale otoka Krka. Acta Adriat. 21 (2), 355-367.

Sket, B., Bole, J., Benović, A., BrancelJ, A., Brglez, J., Čuček, M., Ćurčić, B., Jaklin, A., Karaman, G., Katavić, I., Kerovec, M., Kos, I., Legac, M., Mršić, N., Malej, A., Novak, T., Petkovski, S., Petkovski, T., Polenec, A., Potočnik, F., Pujin, V., Radujković, B., Števčić, Z., Tarman, K., Travizi, A., Velikonja, M., Velkovrh, F., Vidaković, J. \& Zavodnik, D., 1991: Bogastvo in 
raziskanost jugoslovanske favne: nižji nevretenčarji (Metazoa Invertebrata, ex Insecta). Biol. vestn. Ljubljana 39 (1, 2), 37-52.

Zavodnik, D. \& Legac, M., 2012: Prirodna obilježja Puntarske drage na otoku Krku (otok Krk, sjeverni Jadran). In: Prirodoslovna istraživanja riječkog područja II. Prirodoslovni muzej Rijeka, 14-17. 6. 2006. Knjiga priloga, Arko-Pijevac, M. \& Surina, B. (Eds), 253-267.

Zavodnik, D., Legac, M. \& GluhaK, T., 2006: An account of the marine fauna of Pag Island (Adriatic Sea, Croatia). Nat. Croat. 15 (3), 65-107.

Professional papers

Gamulin-Brida, H., Ilijanić, V. \& Legac, M. 1978: Glavonošci - važna komponenta faune i bioloških izvora Jadranskog mora. Morsko ribarstvo 30 (2), 62-69.

Hrs-Brenko, M., Medaković, D. \& Legac, M., 1990: Jestivi školjkaši Jadranskog mora. I. Srčanka jestiva (Cerastoderma glaucum Poiret). Morsko ribarstvo 42 (1), $24-27$.

LeGAC, M., 1968: Rijetki glavonošci Jadrana. More 11 (6), 17.

LegAC, M., 1977: Izložba „Čarobni svijet školjaka“. Vijesti muzeal. konzerv. Hr. 26 (3), 59-62.

LeGAC, M., 1990: Ugroženost pješčanih plaža i faune školjkaša prekomjernim vađenjem pijeska u priobalju otoka Raba. Ekol. Glas. 1 (9-10), 9-14.

Legac, M. \& Hrs-Brenko, M., 1975: Sistematska obrada školjkaša (Bivalvia) i glavonožaca (Cephalopoda) sjevernog Jadrana. Muzeologija 18, 100-105.

Zavodnik, D., Hrs-Brenko, M. \& Legac, M., 1991: Synopsis on the fan shell Pinna nobilis L. in the eastern Adriatic Sea. In: Les Espèces Marines à Protéger en Méditerranée. BoudouresQue, C.F., Avon, M. \& Gravez, V. (Eds). GIS Posidonie publ., Marseille, 1991: 169-178.

Other references used

Alfirević, S., 1968: Results obtained by geological charting of trawling grounds in the channels of Northern Adriatic. Stud. Rev. gen. Fish. Coun. Medit. 37, 15-27.

Alfirević, S., 1980: Sedimentološko kartiranje bentoskih biocenoza u kanalima sjeveroistočnog Jadrana. Geol. vjesnik 32, 15-32.

Alfirević, S., Crnković, D. \& Gamulin-Brida, H., 1969: Problem racionalne eksploatacije škampa, Nephrops norvegicus. Thalassia Jugosl. 5, 5-12.

Amoureux, L., 1979: Campagnes jubilaires du navire de recherches „Vila Velebita“ dans la région nord-est de l'Adriatique. XVII. Annélides polychètes. Thalassia Jugosl. 15, 257-265.

Andrić, M., 2009: Ronjenje u Hrvatskoj. Car Herc d.o.o., Zagreb, 251 pp.

Arko-Pijevac, M., 1998: Prirodoslovni muzej Rijeka - od osnutka do danas. In: Arko-Pijevac, M., Kovačić, M. \& Crnković, D. (Eds), Zbornik 'Prirodoslovna istraživanja riječkog područja', Prirodoslovni muzej Rijeka, Rijeka, 11-52.

Arko-Pijevac, M., 2001: Utemeljenje Prirodoslovnog muzeja Rijeka. Muzeologija 37, 42-57.

Bellan, G., 1976: Contribution á l'étude des Annélides Polychètes de quelques fonds meubles circalittorales des côtes Yougoslaves. Thalassia Jugosl. 12, 391-397.

Carozza, F., 1987: A new bivalve species from Mediterranean: Nuculana illirica spec. nov. (Bivalvia: Palaeotaxodonta: Nuculanoidea). Basteria 1987, 159-161.

CRnković, D., 1970: Prilog biološkoj i ekonomskoj problematici koćarenja u kanalskom području sjeveroistočnog Jadrana. Thalassia Jugosl. 6, 5-90.

Gamulin-Brida, H., Alfirević, S. \& Crnković, D., 1971: Contribution à la biologie de la faune endogée des fonds vaseux de l'Adriatique avec égard spécial à l'espèce Nephrops_norvegicus L. Vie Milieu, suppl. 22, 637-655.

Gauss-GARÁDY, V., 1912: Über die Lebensgeschichte des adriatischen Scampi (Nephrops_norvegicus). Österr. Fisch. Z. 9 (3), 42-44. 9 (4), 61-63. 9 (5), 77-79.

Hemar, E., 2019: In memoriam. Mirjana Legac (1929. -2018.). Hrvatski planinar 110(12), 553.

Kovačić, M., 2006: Ihtiološka zbirka Prirodoslovnog muzeja Rijeka deset godina poslije: veća i bolja? Nat. Croat. 15 (3), 177-185. 
MDC, 1997-2019: Mirjana Legac. Personalni arhiv zaslužnih muzealaca, Muzejski dokumentacijski centar, Zagreb. http://www.mdc.hr/hr/mdc/zbirke-fondovi/arhiv/personalni-arhiv-zasluznihmuzealaca/Legac-Mirjana,146.html (accessed December, 2019).

Murina, G-V.V. \& ZavodniK, D., 1979: Cruises of the research vessel „Vila Velebita“ in the Quarner region of the Adriatic Sea. XVI. Sipuncula. Thalassia Jugosl. 15, 245-255.

Murina, G.-V.V. \& Zavodnik, D., 1985/86: Sipuncula of the Adriatic Sea. Thalassia Jugosl. 21/22, $23-73$.

NATURAL HISTORY MUSEUM RIJEKA (Prirodoslovni muzej Rijeka), 2004: Pravilnik o zaštiti fundusa muzeja, 1-9.

Novosel, M., Bakran-Petricioli, T., Požar-Domac, A., Kružić, P. \& Radić, I., 2002: The benthos of the northern part of the Velebit Channel (Adriatic Sea, Croatia). Nat. Croat. 11 (4), 387-409.

PÉRÈs, J.M. \& GAMulin-BridA, H., 1973: Biološka oceanografija. Bentoska bionomija Jadranskog mora. Školska knjiga, Zagreb, 493 pp.

Pesta, O., 1918: Die Decapodenfauna der Adria. Deuticke, Leipzig-Wien X+500 pp.

Števčić, Z., 1979: Cruises of the research vessel „Vila Velebita“ in the Kvarner region of the Adriatic Sea. XIX. Crustacea Decapoda. Thalassia Jugosl. 15 (3/4), 279-287.

Števčić, Z., 1990: Check-list of the Adriatic decapod Crustacea. Acta Adriat. 31, 183-274.

VILIČıć, D., 2015: Istraživanje mora u Hrvatskoj - sjećanje na jednu generaciju. Hrvatske vode 23 (94), 295-308.

ZavodNIK, D., 1973: Données quantitatives sur les peuplements des fonds vaseux-argileux à Nephrops norvegicus dans la région insulaire de l'Adriatique nord-orientale. Rapp. Comm. int. Mer Médit. $21(9), 599-601$.

ZavodniK, D., 1979: Cruises of the research vessel „Vila Velebita“ in the Kvarner region of the Adriatic Sea. XXI. Benthic investigations. Thalassia Jugosl. 15 (3/4), 313-350.

ZavodnıK, D. \& Kovačıć M., 2000: Index of marine fauna in Rijeka Bay (Adriatic Sea, Croatia). Nat. Croat. 9 (4), 297-378.

Zavodnik, D., Pallaoro, A., Jaklin, A., Kovačić, M. \& Arko-Pijevac, M., 2005: A benthos survey of the Senj Archipelago (North Adriatic Sea, Croatia). Acta Adriat. 46 (Suppl. 2), 3-68.

\section{SUMMARY}

Farewell to Mirjana Legac, marine biologist and museum curator D. Zavodnik

Our colleague and dear friend, Mrs. Mirjana Legac, retired museum advisor of the Natural History Museum in Rijeka, left us forever on November 3, 2018. She was born in 1929, in the picturesque village Baška located on the hilly northeastern Krk Island. Her birthplace determined her love to the natural beauties of the sea, islands, and the north Adriatic highlands (VILIčić, 2015). From the early youth she showed a keen interest in natural sciences. With B.Sc. degree in biology, acquired from the University of Zagreb in 1951, Mrs. Mirjana Legac was in 1958 employed as a museum curator in the Natural History Museum in Rijeka, where she worked until her retirement.

She devoted herself to a life-long study of littoral and shallow-water Adriatic ecosystems, with a special interest on shellfish and cephalopod fauna. The publications resulting from these studies are numerous and present an outstanding contribution to our knowledge on marine life in the Adriatic Sea. Research on the biology and distribution of the Norwegian lobster (Nephrops norvegicus) in the northern part of Croatian Adriatic are example of field work of high quality. Likewise, benthos studies in the insular region of the northeastern 
Adriatic remain a model for meticulous application of field research methodology in the area surveyed. A couple of published results were reviewed in part by the index of Rijeka Bay marine fauna (ZavodniK \& Kovačić, 2000). Engagements in shellfish research have been crowned by a monography on Rijeka Natural History Museum collections (LegAC, 2012).

\section{SAŽETAK}

Oproštaj od Mirjane Legac, morske biologinje i muzealke

D. Zavodnik

Naša kolegica i draga prijateljica gđa Mirjana Legac, umirovljena muzejska savjetnica Prirodoslovnog muzeja u Rijeci, zauvijek nas je napustila 3. studenog 2018. godine. Rođena je godine 1929. u slikovitom mjestašcu Baški na sjeveroistočnom dijelu brdovitog otoka Krka (VILIčć, 2015). Već od ranog djetinjstva su je privlačile prirodne ljepote mora, otoka i brda njezina zavičaja. Bavila se odgonetanjem tajni prirodnih znanosti, naročito biologije. Nakon stjecanja diplome na Prirodoslovno-matematičkom fakultetu Sveučilišta u Zagrebu godine 1951. godine, zaposlila se 1958. kao kustos u riječkom Prirodoslovnom muzeju kojemu je ostala vjerna cijeloga života.

Uz redovna kustoska zaduženja, kolegica Legac se bavila proučavanjem obalnih ekosustava sjevernog Jadrana, posebno školjkaša i glavonožaca te rasprostranjenosti škampa (Nephrops norvegicus). Rezultate svoga rada prikazala je u brojnim znanstvenim izvornim i preglednim te stručnim raspravama. Dio rezultata prikazan je popisom morske faune Riječkog zaljeva (ZAvodNIK \& Kovačıć, 2000). Životna iskustva Mirjaninih istraživanja školjkaša objavljena su u monografskom izdanju Prirodoslovnog muzeja Rijeka (Legac, 2012). 